\title{
Development of a Mobile Tele-Simulation Unit Prototype for Training of Rural and Remote Emergency Health Care Providers
}

\author{
Jennifer Jewer, Adam Dubrowski, Kristopher Hoover, Andrew Smith, Michael Parsons \\ Memorial University of Newfoundland \\ jenniferj@mun.ca, adam.dubrowski@gmail.com, Kristopher.hoover@mun.ca, Andrew.smith@med.mun.ca, \\ mhparsons14@hotmail.com
}

\begin{abstract}
Emergency health care providers in rural and remote areas do not have the same access to training as those in urban areas. This poses a serious challenge to the provision of equitable healthcare delivery. This paper outlines the development of a Mobile TeleSimulation Unit (MTU) prototype to address the challenges of training in rural and remote settings. The goal of the MTU is to increase opportunities for emergency health care providers to attain training remotely. Mobile tele-simulation is a new approach to remote medical training with many clear benefits however one must understand how to develop such a unit and its effectiveness in teaching procedural skills. In this paper, we describe our multidisciplinary mixedmethods approach to develop the MTU using proven theoretical frameworks. We also discuss the developmental challenges, findings on trainee satisfaction and learning outcomes. Initial results are promising and warrant a formal evaluation stage to complete our study.
\end{abstract}

\section{Introduction}

Rural and remote practice of emergency medicine presents unique challenges, particularly when faced with infrequently encountered cases and procedures [1]. These challenges are amplified by the fact that a large proportion of emergency care in rural areas must be provided by physicians who are not emergency medicine specialists or by nurses and nurse practitioners $[1,2]$. This poses a serious challenge to equitable healthcare delivery if patients in rural areas do not have access to comparable levels of emergency care as those in urban centres [3]. Simulation-based medical education (SBME) is a valuable tool in the acquisition and maintenance of knowledge and skills [4,5]; however, simulators are often located in urban centers and they are not easily accessible outside these centers due to geographic, cost and time constraints. Mobile tele-simulation has the potential to overcome these barriers but challenges such as a comfortable learning environment, technical issues and ability to teach desired content via tele-simulation must be addressed. We are developing a Mobile TeleSimulation Unit (MTU) prototype that enables mentors and trainees, emergency health care workers, to connect and access SBME on procedural skills in rural and remote settings. This study aims to obtain a proof of concept regarding the acceptability, feasibility, and effectiveness of the proposed intervention. The goal is to determine whether using this unit, in areas where simulation training would otherwise not be available, is acceptable given the proposed advantages that a MTU can offer in terms of flexibility, convenience and costs. The specific objectives of this project are:

1. Acceptability and feasibility: To gather feedback on the design and function of each iteration of the MTU prototype to incorporate into the finalized MTU.

2. Effectiveness: To examine learning outcomes and assess if the outcomes in the MTU are comparable to face-to-face training.

This study takes place in Newfoundland and Labrador (NL), Canada where 60 percent of the population lives in rural areas. NL has a population of around 500,000 that is geographically dispersed across the province, which is approximately $405,000 \mathrm{~km}^{2}$ or almost one and three quarters the size of Great Britain. NL has a new simulation lab at the medical school in the capital city; however, the geographic dispersion of medical facilities across the province makes it expensive and time consuming and often impractical for trainees to train at the urban simulation centre.

\section{Background}

Mobile tele-simulation is a combination of telesimulation and mobile simulation. Tele-simulation involves using the internet to give trainees access to simulators in a different location. It couples the principles of simulation with remote internet access to teach procedural skills [6]. Tele-simulation has been 
shown to be an effective means of teaching medical skills [7-12]. However, trainees may not have access to simulation equipment or the training environment necessary for tele-simulation.

Mobile simulation, alternatively, enables access to simulation training by bringing necessary equipment, and sometimes even the training environment, directly to the remote teaching site. Mobile simulation can consist of a specialized unit comprising portable simulation equipment that effectively represents a safe, immersive classroom environment for simulation training. For rural areas, or those without access to a dedicated simulation center, mobile simulation is an especially valuable resource for the delivery of medical training [13-18]. However, bringing the mentor, experienced in the subject area and in effective simulation based education and debriefing, to the learner can often prove to be quite expensive.

Since accessibility to both an expert mentor, along with the appropriate training environment and equipment, can be obstacles to simulation training in rural and remote areas, merging the two concepts of tele-simulation and mobile simulation presents an innovative solution. To our knowledge, research on the concurrent application of tele-simulation and mobile simulation to deliver medical training has yet to be conducted.

\section{Description of MTU Prototype}

Using the MTU, short "skills labs" will be delivered remotely to emergency health care providers in rural or remote locations using content developed by mentors experienced in the subject area and in SBME. The MTU would be transported to the location and is designed to require minimal technical support to set up and carry out the training session. Educational content of the modules delivered can be variable and tailored to the needs of the learner. The geographically separated mentor would deliver the skills lab remotely via a live broadcast with two-way video and audio. The importance of a mentor with experience in the clinical environment and with delivering simulation training remotely cannot be underestimated [19]. All sessions would consist of a briefing, simulation scenario and debriefing. Relevant review materials would be sent out to learners prior to each session to allow presession familiarization with key information.

The two main goals for the MTU are increased efficiency and comparable educational effectiveness:

1. More Efficient Training - MTUs would be less expensive than in-person instruction; neither the trainee nor the mentor would need to travel (saving valuable time); MTU would use low cost and off-the-shelf communications technology through a coordinated program of simulation equipment sharing and use of lowfidelity models to effectively deliver educational content.

2. Comparable Effectiveness - Learning outcomes must be comparable to in-person delivery of training and trainees must be satisfied with the training sessions.

\section{Methods}

The iterative development of the MTU prototype was carried out through a mixed-methods approach and with input of a multi-disciplinary team with backgrounds in emergency medicine, clinical simulation, health informatics, engineering and research. To develop the MTU prototype we followed Haji et al.'s [20] adapted Medical Research Council (MRC) framework to develop programs in simulation education for training of health professionals. The MRC emphasizes a theory-based, iterative programmatic approach to designing SBME. The MRC framework (Figure 1) was originally created for development of complex clinical interventions and has been successfully applied in that area [21].

The MRC consists of four cycles of the research process:

Cycle A - Theory and Modelling;

Cycle B - Piloting;

Cycle C - Evaluation, and;

Cycle D - Implementation.

We followed an iterative approach and are currently completing the analysis of the results from Cycle B, piloting. Cycle $\mathrm{C}$ will begin in the near future. The necessary institutional ethics review board approval was obtained before the project began and initial results of this study have been presented at academic conferences [22, 23].

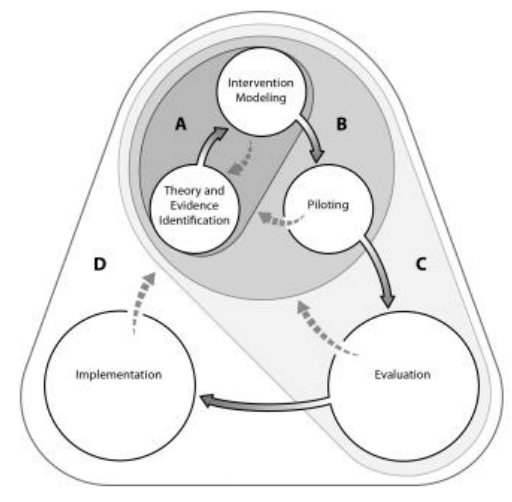

Figure 1 - MRC framework [20] 


\subsection{Cycle A - Theory and Modelling}

We started by identifying the need for improved rural emergency health care providers' access to training. We then set about determining how to address this need and deliver the training remotely. A review of the literature revealed significant research on telemedicine, and selected research on mobile units; however, there was very limited research on mobile tele-simulation units. Using Cristancho et al.'s [24] Aim-FineTune-FollowThrough (AFT) process to guide the design of the MTU prototype we moved through the iterative development process. The AFT process is grounded in learning theory and was developed to aid the development of simulation training programs. The AFT process has been used to successfully design a simulation-based program to train surgeons [25]. In the "Aim" stage of the AFT process we selected the procedural skill to be taught, broke the design into main components, and developed a concise, measurable definition of each component. We then used motor and cognitive modeling diagrams (MCMD) to determine processes, decisions and logic required to complete the components of the MTU prototype on three main areas - comfort, technology and human factors. In the "FineTune" stage we used the Delphi method to collect input from experienced emergency physicians on key design components, important features of mobile tele-simulation and potential applications in teaching and research. We also revised the MCMDs and determined evaluation points and performance measures. In the "FollowThrough" stage we finalized the MCMDs and developed and validated the MTU prototype.

4.1.1. Development of MTU prototype. We designed the MTU prototype to ensure an efficient arrangement and operation of tele-communications and simulation equipment to allow ease of instruction, procedural performance and assessment. Table 1 identifies the design and technical features that guided the design of the MTU prototype.

As the main focus of the study design was to assess educational effectiveness of a mobile tele-simulation unit an inflatable rapid deployment tent was determined to be the most acceptable solution (Figure 2). Vehicle and trailer based units were much more expensive and felt to be impractical at this point. The MTU tent was obtained locally in NL from Dynamic Air Shelters ${ }^{1}$. Its robust construction makes it suitable for transport and deployment in a variety of harsh environmental settings. Table 2 and Figure 3 show an overview of the equipment used in the MTU prototype.

\footnotetext{
${ }^{1}$ https://www.dynamicairshelters.com/
}

4.1.2. Development of training program. We applied the best practices of SBME pedagogy outlined by McGaghie et al. [19], including: feedback, deliberate practice, outcome measurement, simulation fidelity, and skill acquisition and maintenance.

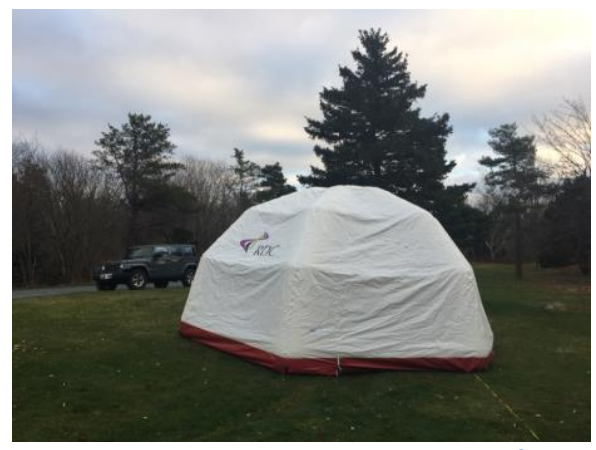

Figure 2-Rapid deployment tent designed to function as the MTU

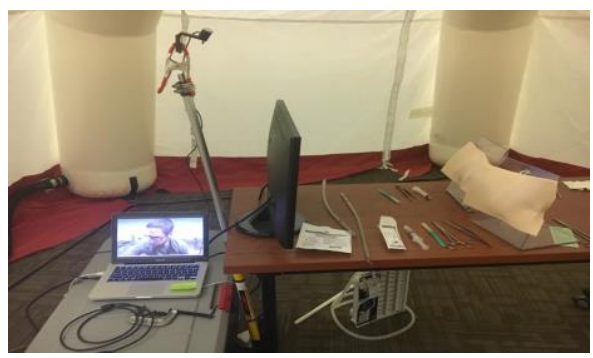

Figure 3 - MTU with simulation setup at the remote site and mentor presence via telecommunication

The approach that we used for the delivery of educational content during the study included presession delivery of background information to the learner followed by hands-on teaching during instructional sessions. The pre-session information consisted of an online New England Journal of Medicine video demonstrating the procedure and providing other important details [26]. At the hands-on sessions, learners would receive guidance and real-time feedback on their performance. Pre and post tests with brief topic related questions were used to assess knowledge on the topic. We also assessed procedural performance skills through learner practice on the low fidelity models. Debriefing is essential to SBME [27], therefore following the four-step model presented by Rudolph et al. [28] we conducted debriefing with provision of relevant feedback. The real-time two-way communication between the mentor and trainees enabled this feedback. We designed the session to allow for deliberate practice which has been found to be an important part of SBME [29]. During the sessions, the trainee is given an opportunity to perform the procedure and receive feedback on their performance with the opportunity to ask questions. 
Table 1 - Features of the MTU prototype

\begin{tabular}{|l|l|}
\hline Feature & Description \\
\hline Physical - Size/Layout of the & Balance portability of unit with available work space \\
\cline { 2 - 2 } MTU & Ability to adapt space to variety of simulation scenarios \\
\hline \multirow{2}{*}{ Technical - } & Real time communications- simple and easy to use \\
\cline { 2 - 2 } & Displays and quality of audio-visual communications \\
\cline { 2 - 2 } & Infrastructure either cell or broadband network \\
\cline { 2 - 2 } & Low cost software communications platform \\
\hline Practical considerations & Efficient heating, ventilation, wiring, lighting, power supply \\
\hline
\end{tabular}

Table 2 - General equipment for setup of the mentor base station and the remote MTU station

\begin{tabular}{|l|l|}
\hline Mentor Side & Remote Trainee \\
\hline$\bullet$ Technology & $\bullet$ MTU Tent \\
- PC with communications software, used the free & $\bullet$ Technology \\
version of VSee communications & - Laptop with communications software (VSee) \\
- Web camera, speaker and microphone & - LCD monitor \\
- Simulation Materials & - Web camera, speaker and microphone \\
- Medical instruments and supplies for procedure & - Portable wireless internet hub \\
including simulated materials & - Simulation Materials \\
- Match the setup to the remote trainee station & - Medical instruments and supplies for procedure \\
\hline
\end{tabular}

Table 3 - Select features of each MTU prototype evaluation sessions

\begin{tabular}{|l|l|l|l|}
\hline & Session A & Session B & Session C \\
\hline Location & $\begin{array}{l}\text { Wilderness Setting } \\
\left(5{ }^{\circ} \mathrm{C}\right)\end{array}$ & $\begin{array}{l}\text { Wilderness Setting } \\
\left(-20^{\circ} \mathrm{C}\right)\end{array}$ & Inside \\
\hline Procedural Skills Covered & Joint reductions & \multicolumn{2}{|c|}{ Tube thoracostomy (chest tube) } \\
\hline No. of Trainees & 35 & 6 & 18 \\
\hline Trainee Background & $\begin{array}{l}\text { Family medicine } \\
\text { residents }\end{array}$ & $\begin{array}{l}\text { Family medicine } \\
\text { residents and nurses }\end{array}$ & Medical students \\
\hline No. of trainees who did procedure before & Did not ask & $3(1-2$ times $)$ & 0 \\
\hline Past exposure to Telemedicine & $30(86 \%)$ & $5(83 \%)$ & $3(17 \%)$ \\
\hline Past exposure to low fidelity SBME & $20(57 \%)$ & $6(100 \%)$ & $17(94 \%)$ \\
\hline
\end{tabular}

Our session was geared toward teaching an important procedural skill, with joint reductions at Session A and tube thoracostomy (chest tube) at Sessions B and C. Joint reductions were taught with trainees doing hands on practice on each other. In contrast, chest tube placement was taught using a low fidelity setup with the use of $3 \mathrm{D}$ printed ribs on a plexiglass stand with low-cost skin and subcutaneous tissue used (Figure 3).

\subsection{Cycle B -Piloting}

Piloting is divided into four sub-phases: (1) establish feasibility and acceptability; (2) clarify uncertainties in the design of the intervention and outcome assessment; (3) identify and design the training protocol for a comparison group, and; (4) address methodological issues. These sub-phases are independent and not completed in any particular order. We held three prototype evaluation sessions to complete these four sub-phases and pilot the MTU prototype. This also involved iteratively applying the AFT process. The descriptions of the sessions are presented in Table 3.

4.2.1. Session A. The purpose of the first session, Session A, was to evaluate the feasibility and acceptability of the MTU and to clarify uncertainties in the design of the intervention. We considered possible barriers to the prototype implementation and addressed technical issues. We also evaluated and documented the set-up and take down of the MTU and all related components, since the MTU needs to be able to be setup by a technician at a remote site. The MTU prototype was set up in a wilderness setting and 35 family medicine residents received training on joint reduction 
as a part of their wilderness training course. Joint reduction was selected because of its relevance to the rural practitioner. The trainees were divided into 4 groups with approximately 9 trainees receiving the training at the same time. Following the format for the curriculum described in section 4.1.2 of this paper, an experienced emergency physician (the mentor) taught the trainees how to reduce an elbow dislocation remotely via a tele-communications link and the trainees had the opportunity to interact with the mentor. There was a camera on the mentor which the trainees could see on the laptop screen in the MTU; there were also two cameras in the MTU so that the mentor could observe the trainees' performance. Since this was our first trial with the MTU, an experienced emergency physician present in the MTU also demonstrated reduction of finger and shoulder dislocations.

Students were asked to fill out a general information survey at the beginning of the session and a design survey at the end of their session. The general information survey collected information on demographics and past-experience with the procedure, SBME and tele-medicine training. The design survey focused on design and telecommunications features of the MTU, and perceptions of learning experiences. The features were rated on a five-point Likert scale from strongly disagree (1) to strongly agree (5).

4.2.2. Session B. Prototype B incorporated feedback from Session A involving family medicine residents at the Wilderness course and also took into consideration the comments of research team members with respect to improvements. The purpose of Session B was to continue to examine the feasibility and acceptability of the MTU and clarify uncertainties in the design of the intervention and outcome assessment.

Session B saw the MTU transported by airplane to Labrador, a more remote Northern region of the province. It was necessary to address challenges of packaging and transport with this deployment. The extreme environment, with its very cold temperatures (minus 20 degrees Celsius), added additional challenges to the effective delivery of our educational content. Chest tube insertion was chosen as the procedure for this session as it was felt to be an important skill for learners and it was amenable to lowfidelity simulation setup and effective demonstration by the remote mentor. Learners were instructed remotely on the completion of a chest tube insertion procedure on 3D printed low-fidelity models following the curriculum described in section 4.1.2. No on-site mentor was present in this session. We reduced the number of trainees receiving training in the MTU in each session from nine to two, acting on feedback from
Session A with respect to learner to instructor ratios. Additionally, due to the lag with two cameras in Session A we decided to use just one camera in Session B.

As with Session A, trainees completed the general information survey before the session and completed the design surveys after the session. Learning outcomes were also evaluated in this session. To measure outcomes we used a combination of trainee response and observational assessments of performance. The design survey was updated to include measures of learning outcomes adapted from the National League for Nursing (NLN) Student Satisfaction and Self-Confidence in Learning scales to measure beliefs and attitudes about learning in simulation [30]. These scales have been widely used and have been found to have sufficient reliability and validity to be used in education research [31]. The trainees were recorded performing the procedure before and after the session and their performance was assessed using a predetermined skills checklist. Additionally, trainees were given a set of procedure specific questions to answer before and after the session, and a set of questions on learning outcomes. These materials were evaluated by an experienced physician to determine if differences existed pre and post session. We found that the extreme cold temperatures presented challenges. The space heater was not able to cope with the minus 20 degrees Celsius temperatures, and some related discomfort was noted by participants. As well, low temperature resulted in compromised seals on the tent components and related slow air leak requiring re-inflation during the sessiona process requiring air blowers and potentially a generator, all leading to significant noise interference.

4.2.3. Session C. This session continued to build upon information gathered from the earlier prototype design cycle. We continued to evaluate the design and function of the MTU but also worked to complete the third and fourth sub-phases of Cycle B, the design of the training protocol for the comparison group and addressing any methodological issues. Because the overall purpose of this MTU prototype is to deliver training comparable to face-to-face training, we designed the training session for the comparison group to be given in this manner. The same procedure was taught (i.e. chest tube), using the same medical instruments, supplies and low-fidelity ribs for setup. The session was given the same amount of time for the face-to-face and tele-medicine groups. The session even took place in the MTU tent to minimize any environmental influencers as compared to Session B, although this round of testing was in a warm environment. Eighteen first and second year medical 
students were the subjects for this session. Three groups of equal sizes were created: the intervention group (tele-medicine), the comparison group (face-toface), and the control group (no training session). Since this is a noninferiority study a control group was needed to confirm that not only is the intervention group not inferior to the comparison group but that both treatments are actually effective [32]. Trainees were randomized to each group based on the order of their reply to request for participation and we delivered the session to two trainees at a time. A third student per group was put in the control cohort and did not receive training (either remote or face-to-face). Instead they worked on solving a game puzzle for 20 minutes and then completed the post- tests and surveys.

Upon arrival at the session the trainees completed the general information and design surveys as in the previous sessions. Learning outcomes of trainees were evaluated using the instruments from the Session B. To evaluate skill maintenance over time the trainees were tested 1-week after the training session using the skill questions and their performance of the procedure was recorded. We also asked if they had performed, witnessed or received training in chest tube insertions in the week prior to doing the retention test.

\section{Results}

Through each successive session the MTU was evaluated on physical design of the unit, function of the telecommunications equipment and overall impression on the utility of the MTU. All trainees completed these questions with the exception of the 6 control and 6 face-to-face trainees in Session $\mathrm{C}$ who did not receive remote training. The trainees' ratings on a scale of 1 (lowest) to 5 (highest) on the design features, tele-communications, and overall satisfaction with the MTU are shown in Figures 4, 5 and 6 respectively. The Appendix shows the means and standard deviations.

As shown in Figure 4, the design features were rated at around 4 or higher for all sessions, except for noise. There were no statistically significant differences between sessions on design features, other than noise level as determined by one-way ANOVA. On noise level there was a statistically significant difference between groups $(F(2,44)=9.795, p=.000)$. A Tukey post hoc test revealed that the noise problem was statistically significantly higher in the B Session $(2.67 \pm 1.211, p=.000)$ than in the A Session $(4.23 \pm$ $.646)$. There was no statistically significant differences between the other sessions $(\mathrm{A}$ and $\mathrm{C}$ Sessions $=.133$ and $\mathrm{B}$ and $\mathrm{C}$ Sessions $=.209$ ). No significant relationships were found for the other features: equipment well-organized $(\mathrm{F}(2,44)=1.311, \mathrm{p}=.280)$, for good lighting $(\mathrm{F}(2,44)=2.113, \mathrm{p}=.133)$, or for adequate space $(\mathrm{F}(2,44)=2.890, \mathrm{p}=.066)$.

As shown in Figure 5, the features of the telecommunications were rated at around 4 or higher for camera set-up for all sessions. There was no statistically significant difference between groups on camera set-up $(\mathrm{F}(2,44)=.042, \mathrm{p}=.959)$. However, the ratings on audio were lower and there was a statistically significant difference between groups on the audio $(F(2,44)=6.131, p=.004)$. A Tukey post hoc test revealed that the audio problem was statistically significantly higher in the B Session (2.83 $\pm 1.169, p=.007)$ than in the A Session (4.09 \pm .853$)$. There was no statistically significant difference between the other sessions (A and C Sessions $=.147$ and $\mathrm{B}$ and $\mathrm{C}$ Session $=.598)$.

Figure 6 shows that the trainees across all sessions rated their level of satisfaction around 4 or higher and indicated that they would recommend the MTU prototype to their colleagues. There was no statistically significant difference between sessions on overall satisfaction with MTU $(\mathrm{F}(2,44)=1.772, \mathrm{p}=.183)$, or for whether they would recommend the MTU to colleagues $(\mathrm{F}(2,44)=2.480, \mathrm{p}=.096)$. The ratings were high across all sessions with ratings on satisfaction in Sessions A (3.90 \pm .746$)$, B (4.00 \pm $0.632)$, and $C(4.50 \pm .548)$. The ratings were also high on whether the trainees would recommend the MTU in Sessions A (4.09 \pm .712$)$, B (4.67 \pm 0.516$)$, and C (4.50 $\pm 0.548)$.

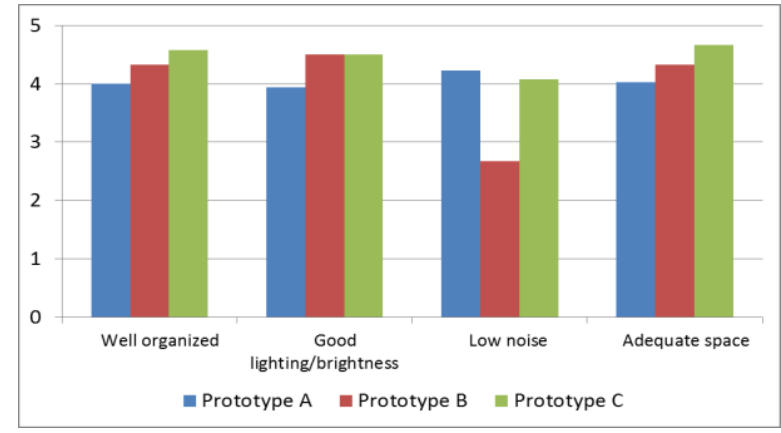

Figure 4 - Feedback on physical MTU design features

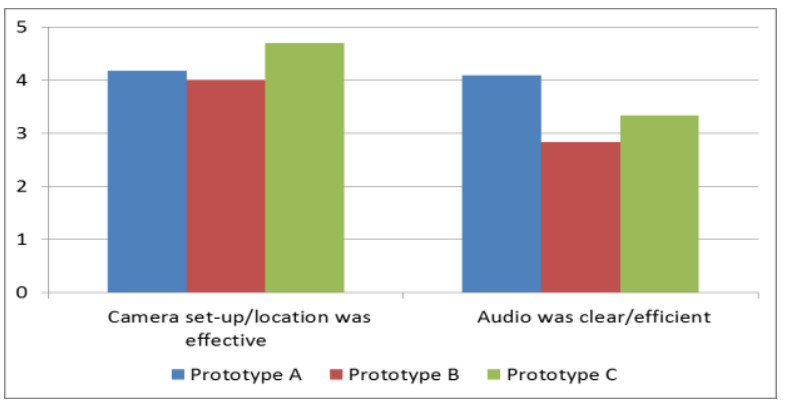

Figure 5 - Function of telecommunications 


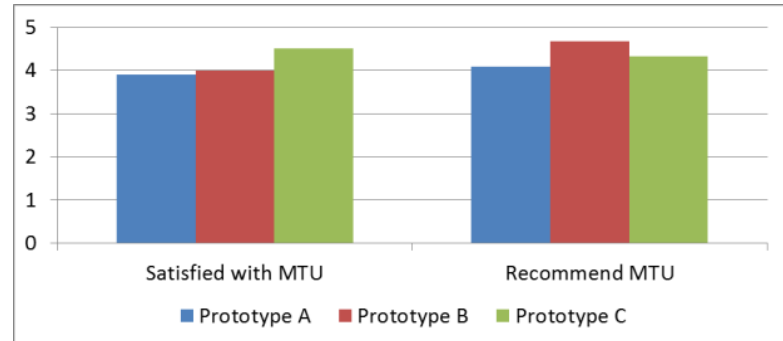

Figure 6 - Overall satisfaction with the MTU

In addition to examining the acceptability and feasibility of the MTU, in Sessions B and C we also examined the effectiveness of the MTU in terms of learning outcomes. We measured beliefs and attitudes about learning as well as objective measures that involved evaluating performance of the procedure and a set of skill questions. Measures for beliefs and attitudes consisted of questions on objectives and information, satisfaction with learning, self-confidence in learning, complexity, cue and feedback/debriefing. The Appendix shows the means and standard deviations. The results in Session B indicated that there was some room for improvement in the training program with the average rating on items ranging from 3.17 to 4.17 on a scale of 1 (lowest) to 5 (highest). We used this feedback to make changes to Session C. For example, we included a video on the procedure in the pre-session materials in Session $\mathrm{C}$ to provide information at the beginning of the session to provide direction and encouragement.

We also performed a preliminary analysis on the impact of the intervention comparing the groups in Session $C$ that received the training remotely versus face-to-face. An independent samples t-test revealed that there were no statistically significant differences between the groups on any of the items except for the items measuring cues. We found that trainees in the face-to-face training reported significantly higher on the cues provided, measured by items: "enough information provided to me during the session" and "cues are appropriate and geared to promote my understanding" ( $4.5 \pm 0.548$ for both items), compared to trainees who received training remotely $(3.83 \pm$ 0.408 on both items), $\mathrm{t}(10)=-2.390, \mathrm{p}=0.038$.

Learning outcomes were assessed using objective measures after Sessions B and C. We have completed the analysis on the trainee completion of the skill questions in Session B. Skill questions were evaluated by an experienced emergency physician. Only four trainees completed the skills test before and after the session so it is not possible to statistically analyze the outcomes, but it was found that the scores on the test increased for all trainees after they completed the training session (Table 4).
Table 4 - Session B pre and post-test skills test results

\begin{tabular}{|l|l|l|}
\hline Trainee & Pre-test & Post-test \\
\hline 1 & $14 / 15(93 \%)$ & $15 / 15(100 \%)$ \\
\hline 2 & $9 / 15(60 \%)$ & $15 / 15(100 \%)$ \\
\hline 3 & $11 / 15(73 \%)$ & $12 / 15(80 \%)$ \\
\hline 4 & $9 / 15(60 \%)$ & $11 / 15(73 \%)$ \\
\hline
\end{tabular}

\section{Discussion}

To our knowledge this is the first report of the development of a MTU for remote training of emergency health care providers. It was helpful to follow the four cycles of the adapted MRC framework to develop the MTU prototype which enabled us to identify challenges in the prototype and to address these challenges iteratively in subsequent prototypes.

Overall, the trainees in each session were satisfied with their experience in the MTU and would recommend the MTU to their colleagues for SBME. Additionally, the design and telecommunication features were rated highly in all sessions except for the noise level in the MTU and the audio quality of the telecommunications equipment. Specifically, issues were noted with the noise and audio during Session B. During this session the extreme cold was associated with air leaks in the MTU structure and required pausing instruction to re-inflate the unit. The other two deployments required no re-inflation. This re-inflation was noisy and we believe it contributed to the lower ratings on satisfaction with the noise and audio. Built in laptop speakers provide adequate audio in most circumstances but external speakers of better quality may be advantageous. One of the key challenges of the development of the prototype was to minimize the costs and keep the MTU easy to set-up with little technical experience, while maximizing the value that the trainees receive. We used off-the-shelf communications software to keep costs low. The challenge with this was that it is developed for high bandwidth; however, the rural or remote locations may not have access to high bandwidth. Setting video quality at low resolution helped with avoiding choppy audio-visual transmission but was associated with compromise of fine detail and made assessment of some components of the skill (eg. suturing) more difficult. Using single camera setups at each of the mentor and remote stations in Session $\mathrm{C}$ helped to solve some of the delays seen in Session B when two cameras were used in the remote station. Further development should look into using purpose-built efficient communications system designed for low bandwidth. 
It was encouraging to find that there were no statistically supported differences in beliefs and attitudes about learning between those who received training remotely versus face-to-face in session $\mathrm{C}$, and that all trainees in Session B performed better on the skills test after the session than they did before the session. These findings are consistent with other studies which have compared SBME with other instruction, and with no intervention [33]. There are still some improvements to be made in the MTU prototype. In particular, we would like to improve the cues provided to trainees during the remote setting since these items were score significantly lower than the cues in the face-to-face session. The noise and audio issues may have played a role here. We will follow-up with trainees to investigate what additional information could be provided to them during the session and how the cues could be improved to ensure they are appropriate and geared to promote trainee understanding of the subject matter. We would also like to improve any items that received a Likert rating less than 4 (see Appendix). We will attempt to improve the information provided before the session, the facilitation of independent problem-solving, provide information in a clear manner to enable the trainee to problem-solve, and the provision of feedback during the session.

The main limitation of the study to this point has been the small sample sizes at each stage of prototype development. Another session is planned to study the MTU with more subjects to enable more meaningful collection and analysis of results. Another limitation is the use of one emergency physician to grade the skills test. We will use at least two independent physicians to grade the skills test and the trainees' performance of the chest tube procedures in the subsequent experiments with consideration of inter-rater reliability.

\section{Next Steps}

The next steps are to evaluate the learning outcomes of Session $\mathrm{C}$ trainees. This will complete Cycle B of the MRC framework. In Cycle $\mathrm{C}$ we will evaluate the educational effectiveness of the MTU by using the MTU with a larger group of medical students so that we will be better able to statistically analyze the results and compare the pre and post-tests on learning outcomes. If we find that the learning outcomes delivered remotely are comparable to face-to-face, then we will proceed with Cycle D, implementing the MTU into broader practice settings. The ultimate goal is the delivery of the simulation training remotely through the use of a larger self-contained vehicle containing simulation equipment necessary for a wider range of scenarios. This will present an opportunity to curb geographic, cost and time barriers to emergency medical education provision in rural and remote areas. Future research will also examine the potential delivery of mobile tele-simulation training to other medical disciplines.

\section{Conclusion}

Following a theory-based approach of the MRC framework and the AFT process has helped us to conduct the iterative development of an MTU prototype targeted to meet the learning needs of emergency health care providers in rural and remote areas. Designing a complex intervention, such as the MTU, pose substantial challenges to investigators; however, the use of the frameworks that harness qualitative and quantitative methods should improve the intervention and the study design and generalizability of results. The MTU prototype has been improved through ongoing evaluation, reflection and redesign. Feedback to ensure a quality learning experience in the MTU has directed key features of physical design, technical performance and training program that have been applied in deployment of the unit in each evaluation session. The MTU prototype appears to be an effective means to make quality simulation training on procedural skills more accessible to emergency health care providers in rural and remote areas. Further evaluation of design and telecommunication features, and learning outcomes will help to determine the full potential of the MTU to address some of the challenges to equitable healthcare delivery.

\section{References}

1.Williams, J. M., Ehrlich, P. F., and Prescott, J. E. "Emergency medical care in rural America", Annals of emergency medicine, 2001; 38(3), 323-327.

2.Casey, M. M., Wholey, D., and Moscovice, I. S. "Rural emergency department staffing and participation in emergency certification and training programs", The Journal of Rural Health, 2008; 24(3), 253-262.

3.Dharmar M, Marcin J, Romano P, et al. Quality of care of children in the emergency department: association with hospital setting and physician training. $\mathrm{J}$ Pediatr. 2008;153:783Y789

4.Issenberg SB, McGaghie WC, Petrusa ER, Lee Gordon D, Scalese RJ, "Features and uses of high-fidelity medical simulations that lead to effective learning: a BEME systematic review", Med Teach, 2005; 27(1):10- 28.

5.Cook DA, Hatala R, Brydges R, Zendejas B, Szostek JH, Wang AT, Erwin PJ, Hamstra SJ. "Technology-enhanced simulation for health professions education: a systematic review and meta-analysis", JAMA. 2011;306(9):978-988.

6.Mikrogianakis, A., Kam, A., Silver, S., Bakanisi, B., Henao, O., Okrainec, A., and Azzie, G. "Telesimulation: an 
innovative and effective tool for teaching novel intraosseous insertion techniques in developing countries", Academic Emergency Medicine, 2011, 18(4), 420-427.

7.Wilkiemeyer M, Pappas TN, Giobbie-Hurder A, et al. "Does resident post graduate year influence the outcomes of inguinal hernia repair?" Ann Surg, 2005;241: 879-84.

8.Scott DJ, Dunnington GL. "The new ACS/APDS skills curriculum: moving the learning curve out of the operating room," J Gastrointest Surg, 2008;12:213-21.

9. Strongwater AM. "Transition to the eighty-hour resident work schedule," J Bone Joint Surg Am, 2003;85:1170-2.

10. Schulman CI, Levi J, Sleeman D, et al. "Are we training our residents to perform open gall bladder and common bile duct operations?" J Surg Res, 2007;142(2): 246-9.

11. Ikeyama, T., Shimizu, N., and Ohta, K. "Low-cost and ready-to-go remote-facilitated simulation-based learning", Simulation in Healthcare, 2012; 7(1), 35-39.

12. Ohta, K., Kurosawa, H., Shiima, Y., Ikeyama, T., Scott, J., Hayes, S., ... and Nishisaki, A. "The Effectiveness of Remote Facilitation in Simulation-Based Pediatric Resuscitation Training for Medical Students", Pediatric emergency care, 2016.

13. Ireland, S., Gray, T., Farrow, N., Danne, P. D., and Flanagan, B. "Rural mobile simulation-based trauma team training-an innovative educational platform," Int Trauma Care, 2006, 16, 6-12.

14. Weinstock, P. H., Kappus, L. J., Garden, A., and Burns, J. P. "Simulation at the point of care: reduced-cost, in situ training via a mobile cart," Pediatric Critical Care Medicine, 2009, 10(2), 176-181.

15. Xafis, V., Babidge, W., Field, J., Altree, M., Marlow, N., and Maddern, G. "The efficacy of laparoscopic skills training in a Mobile Simulation Unit compared with a fixed site: a comparative study", Surgical endoscopy, 2013, 27(7), 2606-2612.

16. Pena, G., Altree, M., Babidge, W., Field, J., Hewett, P., and Maddern, G. "Mobile Simulation Unit: taking simulation to the surgical trainee", ANZ journal of surgery, 2015, 85(5), 339-343.

17. Ullman, E., Kennedy, M., Di Delupis, F. D., Pisanelli, P., Burbui, A. G., Cussen, M., ... and Gensini, G. F. "The Tuscan Mobile Simulation Program: a description of a program for the delivery of in situ simulation training," Internal and emergency medicine, 2016, 11(6), 837-841.

18. Bischof, J. J., Panchal, A. R., Finnegan, G. I., and Terndrup, T. E. "Creation and Validation of a Novel Mobile Simulation Laboratory for High Fidelity, Prehospital, Difficult Airway Simulation", Prehospital and Disaster Medicine, 2016, 1-6.

19. McGaghie, W. C., Issenberg, S. B., Petrusa, E. R., and Scalese, R. J. "A critical review of simulation-based medical education research: 2003-2009," Medical education, 2010, 44(1), 50-63.

20. Haji, F. A., Da Silva, C., Daigle, D. T., and Dubrowski, A. "From bricks to buildings: adapting the medical research council framework to develop programs of research in simulation education and training for the health professions," Simulation in Healthcare, 2014, 9(4), 249259.

21. Campbell M, Fitzpatrick R, Haines A, et al. "Framework for design and evaluation of complex interventions to improve health", BMJ, 2000;321:694Y696.

22. Parsons, M., Wadden, K., Pollard, M., Dubrowski, A., \& Smith, A. "P098: Development and evaluation of a mobile simulation lab with acute care telemedicine support," CJEM, 20146, 18(S1), S111-S111.

23. Parsons, M., Smith, A., Hoover, K., Jewer, J., Noseworthy, S., Pollard, M., . . . Dubrowski, A. "P100: Iterative prototype development of a mobile tele-simulation unit for remote training: An update," CJEM, 2017, 19(S1), S112-S112.

24. Cristancho, S. M., Moussa, F., and Dubrowski, A. "A framework-based approach to designing simulationaugmented surgical education and training programs", The American Journal of Surgery, 2011; 202(3), 344-351.

25. Cristancho, S., Moussa, F., and Dubrowski, A. "Simulation-augmented training program for off-pump coronary artery bypass surgery: developing and validating performance assessments", Surgery, 2012; 151(6), 785795.

26. Dev, S. P., Nascimiento Jr, B., Simone, C., and Chien, V. "Chest-tube insertion", "New England Journal of Medicine", 2007; 357(15), e15.

27. Cheng A, Eppich W, Grant V, Sherbino J, Zendejas B, Cook DA. "Debriefing for technology-enhanced simulation: a systematic review and meta-analysis," Medical Education, 2014 Jul 1;48(7):657-66.

28. Rudolph, J. W., Simon, R., Raemer, D. B., and Eppich, W. J. "Debriefing as formative assessment: closing performance gaps in medical education," Academic Emergency Medicine, 2008; 15(11), 1010-1016.

29. Cordray, D. S., and Pion, G. M. 2006. Treatment strength and integrity: Models and methods. In R. R. Bootzin and P.E. McKnight (Eds.), Strengthening research methodology: Psychological measurement and evaluation , (pp. 103-124). Washington, DC, US: American Psychological Association, xix, 299 pp.

30. National League for Nursing, 2005. Retrieved from: http://www.nln.org/professional-developmentprograms/research/tools-and-instruments/descriptions-ofavailable-instruments

31. Franklin, A. E., Burns, P., \& Lee, C. S. Psychometric testing on the NLN Student Satisfaction and SelfConfidence in Learning, Simulation Design Scale, and Educational Practices Questionnaire using a sample of prelicensure novice nurses. Nurse Education Today, 2014, 34(10), 1298-1304.

32. Greene, C. J., Morland, L. A., Durkalski, V. L., \& Frueh, B. C. "Noninferiority and equivalence designs: issues and implications for mental health research," Journal of traumatic stress, 2008, 21(5), 433-439.

33. Ilgen JS, Sherbino J, Cook DA. "Technology-enhanced Simulation in Emergency Medicine: A Systematic Review and Meta-Analysis," Academic Emergency Medicine. 2013 Feb 1;20(2):117-27. 


\section{Appendix - Mean and standard deviation of MTU characteristics}

\begin{tabular}{|c|c|c|c|c|}
\hline Characteristic & $\begin{array}{l}\text { Prototype A } \\
\text { N=35 }\end{array}$ & $\begin{array}{l}\text { Prototype B } \\
N=6\end{array}$ & $\begin{array}{l}\text { Prototype C } \\
\text { N=6 } \\
\text { (Remote) }\end{array}$ & $\begin{array}{l}\text { Prototype C } \\
\text { N=6 (Face- } \\
\text { to-face) }\end{array}$ \\
\hline \multicolumn{5}{|l|}{ Design features of MTU } \\
\hline Well organized & $4.00(0.594)$ & $4.33(0.516)$ & $4.33(0.816)$ & $4.83(0.408)$ \\
\hline Good lighting/brightness & $3.94(0.873)$ & $4.50(0.548)$ & $4.50(0.548)$ & $4.50(0.548)$ \\
\hline Low noise & $4.23(0.646)$ & $2.67(1.211)$ & $3.5(1.378)$ & $4.67(0.516)$ \\
\hline Adequate space & $3.89(0.867)$ & $4.33(0.516)$ & $4.67(0.516)$ & $4.67(0.516)$ \\
\hline \multicolumn{5}{|l|}{ Function of telecommunications } \\
\hline Camera set-up/location & $4.17(1.465)$ & $4.00(1.095)$ & $4.17(0.408)$ & N/A \\
\hline Audio & $4.09(0.853)$ & $2.83(1.169)$ & $3.33(0.816)$ & N/A \\
\hline Satisfied with MTU & $3.90(0.746)$ & $4.00(0.632)$ & $4.50(0.548)$ & $4.5(0.837)$ \\
\hline Recommend MTU & $4.09(0.712)$ & $4.67(0.516)$ & $4.50(0.548)$ & $4.17(1.169)$ \\
\hline \multicolumn{5}{|l|}{ Objectives and Information } \\
\hline $\begin{array}{l}\text { There is enough information provided before the } \\
\text { session to provide direction and encouragement. }\end{array}$ & & $3.83(1.169)$ & $3.67(0.816)$ & $4.17(1.329)$ \\
\hline My need for help was recognized. & & $3.67(0.816)$ & $4.33(0.516)$ & $4.33(0.516)$ \\
\hline $\begin{array}{l}\text { I felt supported by the teacher's assistance during } \\
\text { the session. }\end{array}$ & & $3.83(0.983)$ & $4.17(0.753)$ & $4.17(0.753)$ \\
\hline Independent problem-solving was facilitated. & & $3.67(1.033)$ & $3.67(0.516)$ & $4.33(0.816)$ \\
\hline $\begin{array}{l}\text { I clearly understood the purpose and objectives of } \\
\text { the session. }\end{array}$ & & $4.17(0.408)$ & $4.33(0.516)$ & $4.50(0.837)$ \\
\hline \multicolumn{5}{|l|}{ Satisfaction with Learning } \\
\hline $\begin{array}{l}\text { The teaching methods used were helpful and } \\
\text { effective. }\end{array}$ & & $4.00(0.632)$ & $4.83(0.408)$ & $4.50(0.548)$ \\
\hline I enjoyed how the teacher taught the session. & & $3.50(1.225)$ & $4.67(0.516)$ & $4.67(0.516)$ \\
\hline \multicolumn{5}{|l|}{ Self-confidence in Learning } \\
\hline $\begin{array}{l}\text { I am confident that I am developing the skills } \\
\text { and obtaining the knowledge needed to } \\
\text { understand this procedure. }\end{array}$ & & $3.17(1.169)$ & $4.17(0.408)$ & $4.33(0.516)$ \\
\hline \multicolumn{5}{|l|}{ Complexity } \\
\hline $\begin{array}{l}\text { The session provided enough information in a } \\
\text { clear matter for me to problem-solve the situation. }\end{array}$ & & $3.83(0.983)$ & $3.67(0.816)$ & $4.17(0.408)$ \\
\hline \multicolumn{5}{|l|}{ Cues } \\
\hline $\begin{array}{l}\text { There is enough information provided to me } \\
\text { during the session. }\end{array}$ & & $3.67(1.033)$ & $3.83(0.408)$ & $4.50(0.548)$ \\
\hline $\begin{array}{l}\text { The cues are appropriate and geared to promote } \\
\text { my understanding. }\end{array}$ & & $3.67(1.033)$ & $3.83(0.408)$ & $4.5(0.548)$ \\
\hline \multicolumn{5}{|l|}{ Feedback/Debriefing } \\
\hline Feedback provided was constructive. & & $3.83(0.753)$ & $3.83(0.408)$ & $4.00(0.894)$ \\
\hline Feedback was provided in a timely manner. & & $3.83(0.983)$ & $3.83(0.408)$ & $3.83(0.753)$ \\
\hline $\begin{array}{l}\text { The session allowed me to analyze my own } \\
\text { behavior and actions. }\end{array}$ & & $3.5(1.049)$ & $4.00(0.632)$ & $4.33(0.816)$ \\
\hline $\begin{array}{l}\text { There are enough opportunities in the session to } \\
\text { find out if I clearly understand the material. }\end{array}$ & & $3.17(1.169)$ & $3.50(0.837)$ & $4.00(0.632)$ \\
\hline $\begin{array}{l}\text { I learn from the comments made by the teacher } \\
\text { before, during, or after the simulation. }\end{array}$ & & $3.5(1.049)$ & $4.17(0.408)$ & $4.17(0.753)$ \\
\hline
\end{tabular}

